\title{
Gyrokinetic microtearing turbulence
}

\author{
H. Doerk, F. Jenko, M. J. Pueschel, and D. R. Hatch \\ Max-Planck-Institut für Plasmaphysik, Boltzmannstr. 2, D-85748 Garching, Germany
}

(Dated: February 23, 2011)

\begin{abstract}
The nonlinear dynamics of microtearing modes in standard tokamak plasmas are investigated by means of $a b$ initio gyrokinetic simulations. The saturation levels of the magnetic field fluctuations can be understood in the framework of a balance between (small poloidal wavenumber) linear drive and small-scale dissipation. The resulting heat transport is dominated by the electron magnetic component, and the transport levels are found to be experimentally relevant. Microtearing modes thus constitute another candidate for explaining turbulent transport in such toroidal systems.
\end{abstract}

It is well established that the cross-field transport of energy and particles in magnetically confined plasmas is generally dominated by turbulent processes. Nevertheless, important aspects of turbulent transport are not well understood at present, including the role of field line stochasticity - although first major results in this area were already achieved several decades ago. In 1973, Stix argued that even minute magnetic perturbations can destroy magnetic surfaces, enhancing the radial electron heat flux.[1] A few years later, several authors suggested that one source of such fluctuations may be electromagnetic microinstabilities like microtearing modes. [2-5] The latter are gyrokinetic analogues of the well-known magnetohydrodynamic tearing modes, driven mainly by electron temperature gradients and giving rise to small-scale magnetic islands which may overlap and "stochasticize" the magnetic field. This discovery was followed by numerous theoretical and computational efforts throughout the 1980s and 1990s. However, no clear physical picture emerged concerning both nonlinear and even various linear effects; in particular, it is not entirely clear from the published analytical work whether stabilizing or destabilizing effects dominate the linear physics of microtearing modes under realistic conditions.

Over the last few years, linear gyrokinetic studies have been undertaken, indicating a role of microtearing modes first in spherical (small aspect ratio) [6-8] and later also in standard (medium aspect ratio) [9-11] tokamak plasmas. Furthermore, it was shown that a semi-analytic transport prediction (using severe simplifications) by Drake and co-workers [12] is consistent with certain experimental results from the NSTX tokamak.[13] Investigations of the nonlinear dynamics of microtearing modes by means of $a b$ initio gyrokinetic simulations would be extremely valuable in this context, but are still missing to date. This gap shall be closed in the present Letter. Below, we will investigate (i) what sets the saturation levels of the magnetic field fluctuations; (ii) in which way the magnetic fluctuation level is linked to the heat transport level; (iii) which kind of physics sets the saturation amplitudes and thus the transport levels in the quasisteady turbulent state - firmly establishing microtearing modes as additional candidates for explaining turbulent transport in (standard) tokamaks.
In the following, we will employ the local (flux-tube) version of the gyrokinetic turbulence code GENE $[14,15]$ to address the nonlinear microtearing problem. Such simulations are extremely demanding, since they require high resolution in nearly all directions of the fivedimensional phase space. One main issue is that microtearing modes tend to produce magnetic perturbations which are fairly extended in the radial direction, while the parallel current and the electrostatic potential tend to exhibit fine radial structures. Convergence studies reveal that (for our present physical parameters) a perpendicular box size of $150 \rho_{i} \times 300 \rho_{i}$ and a grid of $384 \times 64 \times 24 \times 32 \times 16$ points in $\left(x, y, z, v_{\|}, \mu\right)$ space are required. Here, $x, y$, and $z$ correspond, respectively, to the radial, binormal, and parallel direction in fieldaligned coordinates, $v_{\|}$is the parallel velocity, and $\mu$ is the magnetic moment. Furthermore, the observed small growth rates require relatively long simulation times to get good statistics in the saturated turbulent state.

As magnetic geometry, we take the model of a tokamak with concentric circular flux surfaces described in detail in Ref. [16]. As the nominal set of physical parameters we choose an inverse aspect ratio of $\epsilon=0.18$, a magnetic shear of $\hat{s}=1$, and a safety factor of $q=3$. These are realistic values for the outer core region of a medium aspect ratio tokamak. The ion-to-electron mass ratio is chosen to be $m_{i} / m_{e}=1836$, and the ion-to-electron temperature ratio is taken as unity. Of special importance is the electron-ion collision frequency $\nu_{e i}$ (using a linearized Landau-Boltzmann collision operator [15]); its nominal value is assumed to lie in the semicollisional regime: $\nu_{e i}=0.02 v_{t e} / R$, where $v_{t e}=\left(T_{e} / m_{e}\right)^{1 / 2}$ is the electron thermal velocity and $R$ is the major radius. In this case, the mean free path $\lambda_{\mathrm{mfp}}=v_{t e} / \nu_{e i}$ is comparable to, but somewhat larger than the connection length $L_{c}=2 \pi q R$. The nominal value of the electron plasma beta is chosen to be $\beta_{e}=0.6 \%$, and the normalized profile gradients are given by $R / L_{T_{e}}=3.5, R / L_{T_{i}}=0$, and $R / L_{n}=1$ unless stated otherwise.

Let us now turn to the simulation results. In a first step, we will focus on the magnetic fluctuation levels: what sets them, and how do they depend on various plasma parameters? According to a model developed by Drake and co-workers, [12] one should expect a 


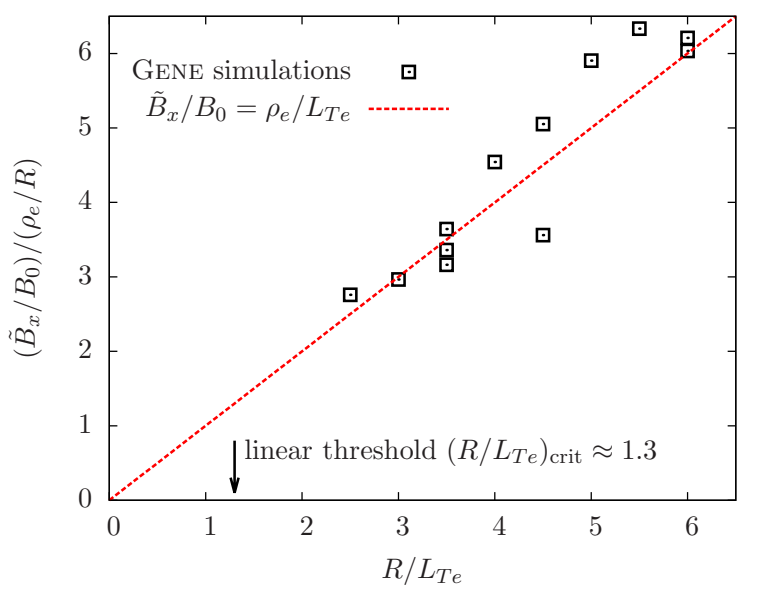

FIG. 1: Microtearing modes saturate at a fluctuation level of $\tilde{B}_{x} / B_{0} \sim \rho_{e} / L_{T_{e}}$. Using the nominal resolution and others, each of which represents a reduction by $1 / 3$ or $1 / 2$ in one of the phase space directions, leads to only moderate scatter.

relative fluctuation amplitude of the magnetic field of $\tilde{B}_{x} / B_{0} \sim \rho_{e} / L_{T_{e}}$, where $\rho_{e}$ is the thermal electron gyroradius. This estimate was obtained in the collisional limit within the framework of a drift-kinetic theory, neglecting parallel dynamics. The results from about a dozen Gene simulations for different values of $R / L_{T_{e}}$ and different numerical resolutions are displayed in Fig. 1. The Drake expectation is shown for comparison as a dashed line. Interestingly, it describes the gyrokinetic simulation results quite well, indicating that the general physical reasoning underlying it might also apply (possibly in some refined form) to the three-dimensional gyrokinetic system. We will return to this important point later. In this context, we would like to note, however, that the magnetic fluctuation amplitude is sensitive to plasma parameters like $\beta_{e}$ or $\nu_{e i}$, as well as various geometrical quantities. As an example, a $\beta_{e}$ scan is shown in Fig. 2. Given that the linear microtearing instability exists only if $\beta_{e}$ exceeds a certain threshold $\left(\sim 10^{-3}\right.$ in the present case), it comes as no surprise that the magnetic fluctuation level increases with increasing $\beta_{e}$. Thus, the Drake formula should only be viewed as a rough estimate which captures some of the main effects but ignores such additional parameter dependencies.

In a second step, we would now like to investigate in which way the magnetic fluctuation level is linked to the associated transport level. Generally, our gyrokinetic simulations show that, while the radial particle flux tends to be very small, the heat flux due to microtearing turbulence is clearly dominated by the magnetic electron contribution $(>80 \%)$. This raises the question if the (magnetic) transport can be described by means of a Rechester-Rosenbluth type [17] ansatz. Since such a model relies on magnetic field stochasticity, we first con-

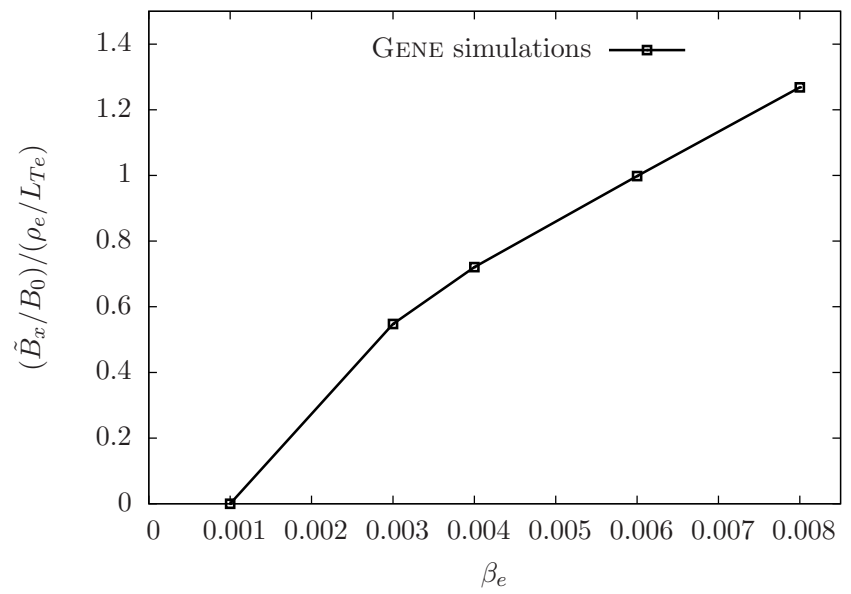

FIG. 2: Dependence of the magnetic field fluctuation amplitude on the plasma parameter $\beta_{e}$ for $R / L_{T_{e}}=4.5$.

firmed that Poincaré plots of static fluctuating field lines support this notion. In Fig. 3, one observes that in the saturated turbulent state, individual microtearing modes create small-scale magnetic islands which may overlap, leading to stochastization. While for weak linear drive $\left(R / L_{T_{e}}=2.5\right.$ where $\left.R / L_{T_{e}}^{\text {crit }} \approx 1.3\right)$, individual magnetic islands survive and the flux surfaces largely remain intact, stronger linear drive $\left(R / L_{T_{e}}=3.5\right)$ induces fully stochastic fields. The resulting radial field line diffusivity can be computed from the GENE simulation data, and it turns out to be well described by the quasilinear expression $D_{M} \sim L_{c}\left(\tilde{B}_{x} / B_{0}\right)^{2}$ (see, e.g., Refs. $[17,18]$ ) once a certain threshold in $\tilde{B}_{x} / B_{0}$ (and thus in $R / L_{T_{e}}$ and $\beta_{e}$ ) is exceeded. For thermal electrons following the fluctuating field lines, the radial heat diffusivity in the weakly collisional regime is then expected to be

$$
\chi_{e}^{\mathrm{em}} \sim v_{t e} L_{c}\left(\tilde{B}_{x} / B_{0}\right)^{2} .
$$

Meanwhile, in the collisional regime, electrons decorrelate from the field lines before they travel a connection length. In this case, the relevant parallel length scale is given by the mean free path $\lambda_{\mathrm{mfp}}=v_{t e} / \nu_{e i}$, modifying Eq. (1). Such a formula was used successfully by Wong [13] to interpret a rather collisional NSTX discharge.

A plot of $\chi_{e}^{\mathrm{em}}$ versus $\tilde{B}_{x} / B_{0}$ for various GENE runs is shown in Fig. 4. The Rechester-Rosenbluth type model from Eq. (1) is displayed for comparison. One finds that the agreement is very good, up to a prefactor of the order of unity. As two prominent outliers (weakly driven cases with $R / L_{T_{e}}=2.5$ or $\beta_{e}=0.003$ ) indicate, however, the model breaks down when the fluctuation amplitudes become small. Here, field line stochasticity is not established, as can be inferred from the Gene data, and the transport level is much smaller than predicted by Eq. (1). In this context, it is worth pointing out that the magnetic transport level can also be linked to certain cross-phase 

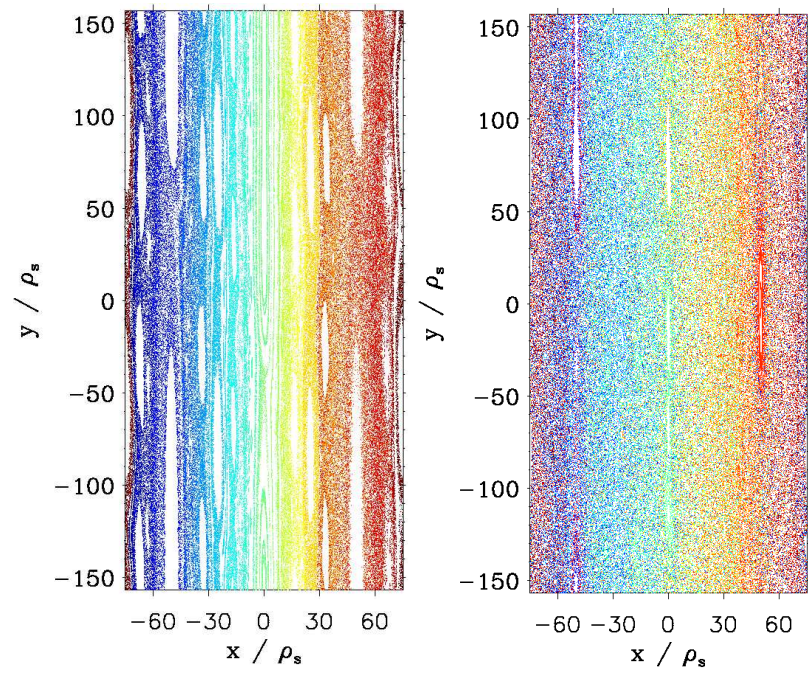

FIG. 3: Poincaré plots of magnetic field lines intersecting the perpendicular plane on the outboard side for weak drive $\left(R / L_{T_{e}}=2.5\right.$, left plot) and stronger drive $\left(R / L_{T_{e}}=3.5\right.$, right plot). The observed features are discussed in the text.

relations as discussed, e.g., in Refs. [19, 20]. One may write $Q_{e}^{\mathrm{em}}=\left\langle\tilde{q}_{e \|} \tilde{B}_{x}\right\rangle / B_{0}$ with

$$
\tilde{q}_{e \|}=-n_{e 0} \chi_{e \|}\left(\frac{\mathrm{d} \tilde{T}_{e \|}}{\mathrm{d} z}+\frac{\tilde{B}_{x}}{B_{0}} \frac{\mathrm{d} \tilde{T}_{e \|}}{\mathrm{d} x}+\frac{\tilde{B}_{x}}{B_{0}} \frac{\mathrm{d} T_{e 0}}{\mathrm{~d} x}\right)
$$

and the parallel electron heat conductivity $\chi_{e} \| v_{t e} L_{c}$. If the third term in Eq. (2) dominates, one recovers Eq. (1). A cross-phase analysis of $\tilde{B}_{x}$ and $\tilde{q}_{e \|} / \tilde{T}_{e \|}$ shows that this is indeed true, provided that the fluctuation amplitudes are sufficiently large.

In this context, we would like to stress that the physics just discussed implies the existence of an effective threshold in the drive (exemplified by $R / L_{T_{e}}$ ) of the microtearing modes. Weak drive leads to small saturation amplitudes of the magnetic islands and a coexistence of stochastic regions with organized structures. Only if the drive exceeds a certain value, can the Chirikov overlap criterion [21] be satisfied, such that the field becomes fully stochastic. Under such conditions, Eq. (1) applies. Thus, there is a certain analogy between this effect and the nonlinear upshift of the effective threshold in ion temperature gradient (ITG) turbulence,[22] although the underlying physics is, of course, completely different.

Interestingly, it is found in our simulations that taking into account equilibrium shear flows, the transport is only reduced by a factor of $\sim 2(\sim 4)$ for shearing rates of a few $(>10)$ times the maximum linear growth rate for the present plasma parameters. This is in contrast to ITG turbulence, where a complete suppression is observed.

In a third and final step, we will now address the question of which kind of physics actually sets the saturation amplitudes in the quasisteady turbulent state. In other words: which are the mechanisms behind the observed

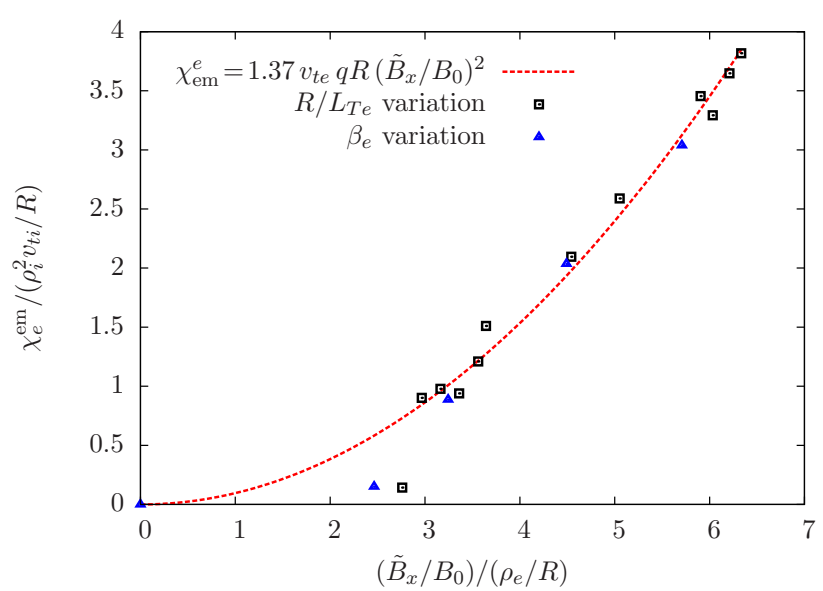

FIG. 4: Electron heat diffusivity as a function of the relative fluctuation level of the magnetic field. The GENE results are described well by the Rechester-Rosenbluth model $\chi_{e}^{\mathrm{em}}=\eta v_{t e} q R\left(\tilde{B}_{x} / B_{0}\right)^{2}$ with $\eta=1.37$. At small fluctuation amplitude, the model breaks down, as explained in the text.

relationship $\tilde{B}_{x} / B_{0} \sim \rho_{e} / L_{T_{e}}$ ? We get some first hints by inspecting the time-averaged electron heat flux spectrum for one of our runs. As is shown in Fig. 5, the linear growth rate spectrum drops to zero at $k_{y} \rho_{i} \sim 0.2$ while a substantial fraction of the transport is driven at much higher wavenumbers. This is in noticable contrast to ITG or trapped electron mode driven microturbulence (see, e.g., Refs. $[23,24])$ and demonstrates that microtearing modes are able to transfer free energy to small perpendicular scales quite efficiently. We also show a spectrum of free energy sources/sinks [25] to demonstrate that (only) the small scales act as a net sink.

To understand this aspect better, it is helpful to recall that individual microtearing modes have a peculiar mode structure. In particular, the electrostatic potential $\Phi$ is highly anisotropic in the perpendicular plane: fine radial scales are present at rather long wavelengths in the $y$ direction. In Fourier space, the same feature is expressed by the fact that at a single $k_{y} \rho_{i} \sim 0.1$, a microtearing mode involves a large number of $k_{x}$ modes which are coupled via the parallel boundary condition. [26] Once the mode amplitude is large enough to allow for nonlinear dynamics, each mode is able to interact with other modes and itself, quickly spreading free energy in wavenumber space and exciting high- $k$ modes which are linearly stable. Performing a secondary instability analysis of the self-coupling process, one finds that modes at twice the value of $k_{y}$ grow linearly with time at a rate of several $v_{t i} / R$ times the square of the primary mode's (normalized) amplitude $\Phi_{p}$. Thus, such transfer processes are comparable to the linear growth rates of about $0.1 v_{t i} / R$ for $\Phi_{p} \sim 0.1$. The observed linear growth rates are comparable to these values, supporting the notion that the nonlinear interactions tend to establish perpendicular 


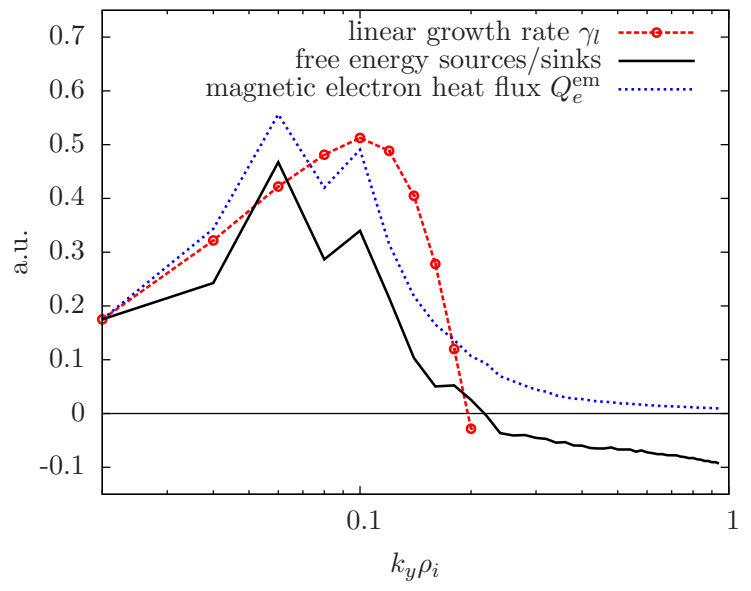

FIG. 5: Time-averaged (magnetic) electron heat flux spectrum in $k_{y}$ space for $R / L_{T_{e}}=3.5$. The linear growth rate spectrum is shown for comparison. Net free energy dissipation is found at $k_{y} \rho_{i}>0.2$.

small-scale isotropy in the system by enhancing high- $k_{y}$ fluctuations - and damping high- $k_{x}$ fluctuations.

Although the mode couplings just described also create zonal flows and fields,[27] the latter are not decisive in this context as can be shown by zeroing them out: this test changes the transport level only by up to about $20 \%$. Instead, the key is the observed strong transfer of free energy to small perpendicular scales. It is evident that free energy pumped into the system via linear drive terms at some rate must be dissipated at the same rate to obtain a saturated turbulence state. Thus, we may assume a balance between the maximum linear growth rates $\gamma_{l}^{\max }$ and the nonlinear damping rates $\gamma_{n l}$, in the same spirit as, e.g., Refs. [12, 28, 29]. In the present case, one may want to model the latter by $\gamma_{n l}=\chi_{e}^{\mathrm{em}} k_{\text {diss }}^{2}$ in terms of Eq. (1) and a typical perpendicular dissipation scale $k_{\text {diss }} \gtrsim 0.2 / \rho_{i}$ as long as field line stochasticity prevails. Using an expression like $\gamma_{l}^{\max } \propto\left(R / L_{T_{e}}\right)^{2} v_{t i} / R$, the Drake estimate, $\tilde{B}_{x} / B_{0} \sim \rho_{e} / L_{T_{e}}$, could be recovered. However, our linear simulations rather suggest something close to an offset-linear dependence of $\gamma_{l}^{\max }$ on $R / L_{T_{e}}$, where both the zero crossing and the slope depend on various plasma parameters (like $\beta_{e}$ ) in a nontrivial fashion. Thus, the relation found in Fig. 1 is not universal, and one should take this fact into account when trying to predict microtearing-induced transport.

In summary, by means of a series of (computationally quite challenging) gyrokinetic simulations of microtearing turbulence in axisymmetric toroidal geometry, the role of field line breaking in the context of turbulent transport was investigated. In particular, it was shown that the heat transport - which is dominated by the electron magnetic component - is linked to the magnetic fluctuation level via a Rechester-Rosenbluth type relation, and that the saturation levels of the magnetic field fluctuations can be viewed in light of a balance between large-scale drive and small-scale dissipation. Moreover, it was demonstrated that the Chirikov criterion for island overlap implies the existence of a threshold in the drive strength, and therefore a nonlinear upshift of the critical electron temperature gradient. Our simulations yield typical electron heat diffusivities of up to a few $\mathrm{m}^{2} / \mathrm{s}$, establishing microtearing modes as additional candidates for explaining turbulent transport in standard tokamaks.

\section{Acknowledgements}

We greatfully acknowledge fruitful discussions with D. Told, T. Görler, F. Merz, and C. M. Roach. The results were obtained with the help of high performance computing resources provided on the HPC-FF system at Forschungszentrum Jülich, Germany.

[1] T. H. Stix, Phys. Rev. Lett. 30, 833 (1973)

[2] R. D. Hazeltine et al., Phys. Fluids 18, 1778 (1975)

[3] J. F. Drake and Y. C. Lee, Phys. Rev. Lett. 39, 453 (1977)

[4] L. Chen et al., Phys. Rev. Lett. 39, 460 (1977)

[5] J. D. Callen, Phys. Rev. Lett. 39, 1540 (1977)

[6] M. Kotschenreuther et al., Nucl. Fusion 40, 677 (2000)

[7] D. J. Applegate et al., Phys. Plasmas 11, 5085 (2004)

[8] C. M. Roach et al., Plasma Phys. Control. Fusion 47, B323 (2005)

[9] D. J. Applegate et al., Plasma Phys. Control. Fusion 49, 1113 (2007)

[10] L. Vermare et al., J. Phys.: Conf. Ser. 123, 012040 (2008)

[11] D. Told et al., Phys. Plasmas 15, 102306 (2008)

[12] J. F. Drake et al., Phys. Rev. Lett. 44, 994 (1980)

[13] K. L. Wong et al., Phys. Rev. Lett. 99, 135003 (2007)

[14] F. Jenko et al., Phys. Plasmas 7, 1904 (2000)

[15] F. Merz, Ph.D. thesis, Universität Münster, 2009

[16] X. Lapillonne et al., Phys. Plasmas 16, 032308 (2009)

[17] A. B. Rechester and M. N. Rosenbluth, Phys. Rev. Lett. 40, 38 (1978)

[18] H. E. Mynick and J. A. Krommes, Phys. Fluids 23, 1229 (1980)

[19] F. Jenko and W. Dorland, Plasma Phys. Control. Fusion 43, A141 (2001)

[20] M. J. Pueschel et al., Phys. Plasmas 15, 102310 (2008)

[21] B. V. Chirikov, Phys. Rep. 52, 263 (1979)

[22] A. M. Dimits et al., Phys. Plasmas 7, 969 (2000)

[23] F. Jenko et al., Plasma Phys. Control. Fusion 47, B195 (2005)

[24] T. Dannert and F. Jenko, Phys. Plasmas 12, 072309 (2005)

[25] H. Sugama et al., Phys. Plasmas 3, 2379 (1996)

[26] M. A. Beer et al., Phys. Plasmas 2, 2687 (1995)

[27] F. Jenko and A. Kendl, Phys. Plasmas 9, 4103 (2002)

[28] W. Dorland et al., Phys. Rev. Lett. 85, 5579 (2000)

[29] F. Merz and F. Jenko, Phys. Rev. Lett. 100, 035005 (2008) 